\title{
Sperm epigenetic profile and risk of cancer
}

\author{
Artur Wdowiak' \\ ${ }^{1}$ Diagnostic Techniques Unit, Faculty of Nursing and Health Sciences, Medical University, Lublin, Poland \\ Wdowiak A. Sperm epigenetic profile and risk of cancer. J Pre-Clin Clin Res. 2014; 8(2): 67-70. doi: 10.5604/18982395.1135652
}

\begin{abstract}
Introduction and objective. The integrity, stability and composition of sperm chromatin are of great importance in the fertilizing potential of male gametes and their capacity to support normal embryonic development. In this study, the author presents the current state of knowledge about the sperm epigenetic profile and risk of cancer.

Abbreviated description of the state of knowledge. The obtaining of pregnancy and the state of health of the baby depends on the quality of the genetic material of both the female and the male. Health behaviours and environmental factors directly affect the quality of sperm, as well as the human egg cell and, consequently, on the reproductive capabilities, the course of pregnancy and the state of the newborn. There exist two thoroughly investigated epigenetic modifications: DNA methylation and histone modifications. The process of DNA methylation can be also a fundamental factor contributing to the development of cancer, where epigenotype undergoes significant modifications. When considering numerous DNA aberrations in the male gamete, the most commonly encountered is DNA fragmentation, particularly in infertile subjects. Surprisingly, an intracytoplasmatic sperm injection study of mice oocytes, using spermatozoa with a high DNA Fragmentation Index (DFI), revealed that a considerable percentage of adults born as a result of this method, showed a significant increase in the incidence of abnormal behavioural tests, malformations, cancer and signs of premature aging.

Summary. The issue of assisted procreation raises the need to look for an appropriate treatment for males with sperm chromatin abnormalities. As a result, the fight against smoking addiction becomes the obvious necessity. Moreover, the reasonable solution nowadays seems to be supplementation with micronutrients and folic acid. It has been proved that the process of DNA fragmentation is a phenomenon that intensifies over time. Therefore, there should be a pursuance for, as close as possible, to the moment of ejaculation, application of semen to reproductive techniques. Finally, epigenetic changes are suspected of being one of the factors responsible for the deterioration of male sperm parameters observed in recent decades.
\end{abstract}

\section{Key words}

sperm, DNA damage, epigenetics, methylation, cancer

\section{INTRODUCTION}

The integrity, stability and composition of sperm chromatin are of great importance for the fertilizing potential of male gametes and their capacity to support normal embryonic development [1]. The obtaining of pregnancy and the state of health of the baby depends on the quality of the genetic material of both the female and the male $[2,3]$.

When taking into account the precise transmission of genetic data, the requirements regarding sperm DNA is to preserve its integrity [4]. The reason for that is the fact of possessing a highly compact and complex structure, as well as being adept at decondensation - these qualities must occur for the spermatozoon to be considered fertile. Furthermore, any flaws regarding sperm chromatin or DNA impairments may cause male infertility. Infertile males exhibit such characteristics as nuclear modifications - an atypical chromatin structure, chromosomes with microdeletions, aneuploidies and DNA strand breaks [5].

When considering numerous DNA aberrations in the male gamete, the most commonly encountered is DNA fragmentation, particularly in infertile subjects. Moreover, evidence has been presented regarding sperm comprising fragmented DNA, outlining its features of being alive, capable of movement, morphologically regular, as well as being able to inseminate an oocyte. Additionally, the oocyte's ability

Address for correspondence: Artur Wdowiak, Diagnostic Techniques Unit, Faculty of Nursing and Health Sciences, Medical University, Lublin, Poland

E-mail: wdowiakartur@gmail.com

Received: 09 October 2014; accepted: 16 October 2014 to repair damaged DNA has been proved; nevertheless, the extent of this process depends on the type of DNA impairment existing in the semen, as well as the quality of the oocyte. Therefore, it is essential to become familiar with the feasible repercussions of sperm DNA fragmentation (SDF) concerning embryo development, implantation, pregnancy outcome, and the health of progeny conceived naturally and supplemented by assisted reproductive technology (ART) $[4,6]$.

Other anomalies which can be detected in sperm chromatin using a high-magnification system, are sperm vacuoles. This abnormality occurs frequently, often multiplies, preferentially anterior, and sperm vacuoles and sperm chromatin immaturity have been associated particularly with large vacuoles. However, its clinical adoption remains unclear in the fields of male infertility diagnosis and assisted reproduction techniques (ARTs) [7]. Nonetheless currently, only one indication regarding the types of DNA impairments existing in human spermatozoa has been uncovered: the high dominance of oxidative base lesions identified among tested subjects. However, the process connected with generating such stress still remains unexplored [8].

State of knowledge. The distinctive features of a genome include genetic variations accountable for the assortment of living beings and their tendency to developing certain diseases. These variations make it difficult to predict the way they are being transmitted from one person to another. As a result, one gene can be articulated in numerous ways in different cells of a single human being. However, there exists 
a way to mediate the mentioned discrepancies in cells or in a single person, by using genetic and/or epigenetic effects. This mediation includes a chemical alteration of DNA as well as its related proteins. These actions may influence cells causing them to remember past contacts with environmental challenges or facilitate genetic effects [9].

Furthermore, there exist two thoroughly investigated epigenetic modifications: DNA methylation and histone modifications. The first, DNA methylation, is an inheritable epigenetic modification, directing gene manifestation and genome stability. However, the role played in gene regulation by DNA methylation may be both passive and active $[10,11]$. Methylation of DNA is a common signalling tool used by cells to place genes in the passive role, a process achieved by the addition of a methyl group prevenient from the $S$-adenosylmethionine (SAM) donor, to the carbon 5 position of cytosines. There exist two different methods by which the regulation is achieved by employing DNA methylation. The first includes physical impediment of the binding of transcription factors to the promoter regions. The second regulation method of DNA methylation involves the process of employing methyl-CpGbinding domain(MBD) proteins, which recruit additional chromatin remodeling factors such as histone deacetylases (HDACs) to construct a compact silent structure [10].

In addition to the importance of DNA methylation in the epigenetic control of gene expression, differential histone tail modifications, such as methylation, phosphorylation, acetylation, and ubiquitinylation, are also key regulators of chromatin states and are referred to as the histone code [12].

The process of DNA methylation can be also a fundamental factor contributing to the development of cancer, where epigenotype undergoes significant modifications. There is the possibility of progressing modification regarding DNA methylation leading to the development of cancer: hypermethylation of $\mathrm{CpG}$ islands, hypermethylation of genes normally methylated $[13,14]$ (global demethylation of genes and local one in promoter genes), transition 5-methylcytosine to thymine, as well as methylation 'non-CpG'. Therefore, abnormal methylation results in changeable levels of gene expression, manifested by instability of the chromosomes and simultaneously stimulates tumour growth [15].

The paternal genome is thought to be crucial in the case of regular growth concerning extraembryonic tissues, whereas embryo development is conditioned by the maternal genome.

All cells, except viruses and spermatozoa, possess a variety of enzymatic mechanisms for repairing damaged DNA. The genetic material of the sperm is formed during spermatogenesis, when DNA methylation and histone modifications are subjected to dynamic changes throughout this process, by means of specialized enzymes. This process, concerned with modifying sperm chromatin, is considered to be complex as well as a remarkable [16]. Chromatin structure undergoes multifaceted morphological and biochemical alterations throughout the synchronized movement of proteins in and out of the nucleus, to generate a distinctive, highly compacted matrix. Additionally, this astounding transformation takes place without the presence of de novo gene transcription. Therefore, spermatogenesis appears to be an object lesson regarding the possibility of control over biological processes by means of a structured translation of the preexisting mRNA species [17].

Taking under consideration the character of protamine connection to DNA and the results regarding the synthesis, as well as assimilation of protamines into spermatid chromatin, the prevailing deduction is that proteins may perform a number of functions. Therefore, DNA is protected from any physical or chemical impairment when the chromatin is unable to do so, consolidating the genomic material to produce a minute, more hydrodynamically-formed cell [18]. DNA repair systems are known to be present in oocytes, having their activity influenced in various ways: genetic and environmental $[19,20]$.

Moreover, endogenous and exogenous DNA mutilations resulting from meiotic recombination, the influence of $U V$ and X-irradiation or the aftermath of mutagenic chemicals, could be revamped by the vertebrate oocyte. This has been proved in numerous in vivo and in vitro systems. However, the oocyte exhibits less healing ability and/or is more sensitive to DNA deteriorating agents previously and later to the dictyate stage of meiosis. In addition, the epigenetic aspects connected with the expression of genetic liabilities arising in oocytes were disregarded in the past. Dictyate oocytes are more likely to display non-disjunctional events, a phase that can be also characterized by oxygen deficiency, perturbations of microtubular structure by temperature and other causes, which may lead to disastrous cytogenetic outcomes. Beside these features, the dictyate stage of meiosis is considered as a resistant resting period $[20,21]$.

When taking into consideration epigenetic programming, the process takes place during embryonic expansion in a gender-specific manner. The imprint of the male germ line is followed by gender determination. This epigenetic programming is susceptible to environmental influences which are able to influence not only the developing scion, but also the subsequent generation. Furthermore, contact with an endocrine disruptor (i.e. vinclozolin or methoxychlor) during embryonic gonadal gender determination can modify the male germ-line epigenetics (e.g. DNA methylation). When considering results of the alteration of DNA methylation in the germ during the epigenetic programming, they include the transfer of transgenerational adult onset conditions, such as spermatogenic defects, prostate disease, kidney disease, as well as cancer. This environmental influence regarding deteriorating of the germ line and to stimulate a transgenerational disease state, has substantial inferences for evolutionary biology and disease etiology [22]. Additionally, authors claim that the origin of $80 \%$ of de novo structural chromosome aberrations in humans is of paternal origin [23], for instance, if DNA damage involved an oncogene, the result would be an increased risk of cancer in the offspring.

Surprisingly, an intracytoplasmatic sperm injection study of mice oocytes, using spermatozoa with a high DNA Fragmentation Index (DFI), revealed that a considerable percentage of adults born as a result of this method, showed a significant increase in the incidence of abnormal behavioural tests, malformations, cancer and signs of premature aging. Anatomo-pathological analysis of the animals at 16 months of age revealed enlarged organs and an increased number of pathologies $33 \%$ of the ICSI (intracytoplasmic sperm injection) produced CD1 females developed solid tumours in the lungs or skin on the back or neck). Furthermore, postmortem anatomical and histological findings indicated that ICSI using spermatozoa with fragmented DNA led to a significant increase in the number of tumours [24].

A concern emerged from studies conducted in smokers regarding an increased risk of childhood cancer observed 
in the offspring of males with a high proportion of sperm with fragmented DNA in their semen. The study in question revealed that the children of these males, whose ejaculates were under oxidative stress [25] and characterized by a high level of chromatin fragmentation, are 4 - 5 times more likely to develop cancer in childhood than the children of nonsmoking fathers [26]. Another study has demonstrated that $15 \%$ of all childhood cancers are directly attributable to paternal smoking [27]. These studies suggest that there may be a link between sperm DNA damage and the subsequent development of childhood diseases.

A study by Wdowiak A et al. demonstrated a considerable influence of passive smoking on the quality of human embryos obtained during in vitro procedures [28].

Radiation induces phenotypic and genotypic alterations in the progeny of treated males. Such genotypic alterations may predispose the progeny of irradiated parents to an increased risk of genetic diseases, infertility, or cancer [21, 29, 30]. Moreover, the consequences of sperm chromatin damage are not limited to the progeny of males exposed to the toxic agent, and several future generations can be affected.

On the other hand, the distinct possibility that DNA repair in oocytes may be enabled by radiation damage is inferred in a paper by Fritz-Niggli and Schaeppi-Buech [19]. An adaptive response to the effects of low doses of X-rays (0.02 Gy prior to $2 \mathrm{~Gy}$ ) was observed in Drosophila melanogaster oocytes, and suggested the existence of 'a repair stimulating effect' of low doses for both the repair-deficient strains, as well as for the highly radiosensitive mature oocytes. This process should not be in opposition to the mechanism in bacteria where DNA damage is responsible for the inactivation of DNA repair repressor molecules.

\section{CONCLUSIONS}

The issue of assisted procreation raises the need to look for an appropriate treatment for males with sperm chromatin abnormalities. As a result, the fight against smoking addiction becomes the obvious necessity. Moreover, the reasonable solution nowadays seems to be supplementation with micronutrients and folic acid [31]. In contrast, there should be also taken into account the negative effects regarding nutritional folate supplementation. According to information originating from mandatory folate fortification of foods in North America in the late 1990s, the supplement not only influenced the targeted neural tube defects in pregnant females, but unfortunately contributed to a concurrent increase in the incidence of colorectal cancer [32]. Additionally, an issue should be raised regarding the possibility of anomalous methylation in sperm that might be transmitted to the offspring in the case of infertile males being supplemented with high doses of folate. This concern demands further studies.

Furthermore, it has been proved that the process of DNA fragmentation is a phenomenon that intensifies over time. Therefore, there should be a pursuance for, as close as possible to the moment of ejaculation, application of semen to reproductive techniques.

The interval between the ejaculation, its preparation, and the microinjection may vary depending on the individual situation. It is common knowledge that donating semen takes a different amount of time for various patients, whereas the microinjection must be performed at the precise moment. Additionally, the research by Pons et al. has proved that DFI also depends on sexual abstinence (the shorter it is, the smaller the DFI and sperm density). The most common sperm selection methods used in ART are 'swim-up' and double-layer gradient centrifugation methods. The period of time concerning centrifugation of the sample depends on the consistency of semen. Additionally, the final result of the DFI just before the microinjection depends on many factors, and may differ from the one obtained just after ejaculation. It is known that the method of preparation depends on the quality of semen. The literature on the subjects provides reports regarding the dependence of the final degree of DNA fragmentation on the method of sperm preparation. Alvarez Sedó et al. [33] evaluated a significant increase of sperm DNA fragmentation taking place after gradient centrifugation or 'swim-up'. In their study, the sperm DNA fragmentation considerably decreased after the centrifugation gradient, regardless of the initial levels of the sample.

Samples with a high DFI were more susceptible to a significant increase in DNA fragmentation over time, with similar increases being observed over time for samples that were incubated in HA (hyaluronic acid) or PVP (polyvinylpyrrolidone). Moreover, various methods of preparation, as well as sperm numerous incubation times, can be influenced by the fact that when used in the seed employed during microinjection will have a different DNA fragmentation than the one straight after being obtained. As it is known that the main cause of damage to the integrity of sperm chromatin is oxidative stress, consequently, the composition of the medium for the preparation may alter the balance in the semen oxydo- reduction system, and the process of preparation is used for selecting the best sperm; therefore, selection will lead to choosing those with less severe fragmentation.

In the case of the intra-uterine insemination cycle, ovulation is induced by providing HCG, an optimal time to perform the procedure is thought to be the between 39 - 41 hours after the injection. However, unknown in this case is the exact time at which the sperm migrate into the bulb fallopian tube. Taking into account the effect of time on the dynamics of DNA fragmentation, it can be assumed that the least damage to sperm chromatin should occur when the resulting pregnancy is an outcome of intra-uterine insemination conducted in the most appropriate time, using the correct method of preparation. It can be predicted that as a result of expectations of sperm in the vaginal canal, which takes place during natural conception, over time, the DNA will undergo deterioration. Therefore, the conclusion could be drawn that healthier children are born as a result of pregnancies obtained by properly conducted intra-uterine insemination, than by natural conception. However, relevant studies need to be conducted to prove this hypothesis.

In the literature, the application of ICSI is associated with an increased incidence of genetic abnormalities in fetuses; however, it needs to be remembered that this method is recommended only in selected cases of male infertility, which are mainly caused by DNA abnormalities, and fertilization cannot be achieved by another method.

Finally, epigenetic changes are suspected to be one of the factors responsible for the deterioration of male sperm parameters observed in recent decades. 


\section{REFERENCES}

1.Albertini DF. Confronting the fissions and fusions of human fertilization. J Assist Reprod Genet. 2014; 31(5): 507-508.

2. Nel-Themaat L, Nagy ZP. A review of the promises and pitfalls of oocyte and embryo metabolomics. Placenta. 2011; 32: 257-263

3. Whitelaw N, Bhattacharya S, Hoad G, Horgan GW, Hamilton M, Haggarty P. Epigenetic status in the offspring of spontaneous and assisted conception. Hum Reprod. 2014; 5(8).

4.Erenpreiss J, Spano M, Erenpreisa J, et al. Sperm chromatin structure and male fertility: biological and clinical aspects. Asian J Androl 2006; 8: 11-29.

5. Hofmann N, Hilscher B.Use of aniline blue to assess chromatin condensation in morphologically normal spermatozoa in normal and infertile men. Hum Reprod. 1991; 6(7): 979-982.

6. Tamburrino L, Marchiani S, Montoya M, Elia Marino F, Natali I, et al. Mechanisms and clinical correlates of sperm DNA damage. Asian J Androl. 2012; 14(1): 24-31.

7.Perdrix A, Rives N. Motile sperm organelle morphology examination (MSOME) and sperm head vacuoles: state of the art in 2013. Hum Reprod Update. 2013; 19(5): 527-541.

8. Tremellen K. Oxidative stress and male infertility - a clinical perspective. Human Reproduction Update 2008; 14: 243-258.

9. Kurus M, Karakaya C, Karalok MH, To G, Johnson J. The control of oocyte survival by intrinsic and extrinsic factors. Adv Exp Med Biol. 2013; 761: 7-18.

10. Bird A. The essentials of DNA methylation. Cell. 1992; 70: 5-8.

11. Mukhopadhyay P, Rezzoug F, Kaikus J, Greene RM, Pisano MM. Alcohol modulates expression of DNA methyltranferases and methyl CpG-/CpG domain- binding proteins in murine embryonic fibroblasts. Reproduct Toxicol. 2013; 1: 1-19.

12. Jenuwein T, Allis CD. Translating the histone code. Science. 2001; 293: 1074-1080.

13. Jabłońska J, Jesionek-Kupnicka D. Zmiany epigentyczne w nowotworach. Onkologia Polska 2004; 7(4): 181-185 (in Polish).

14. Salozhin SV, Prokhorchuk EB, Georgiev GP. Methylation of DNA-One of the major epigenetic markers. Biochemistry (Moscow) 2005; 70(5): 525-532.

15. Sulewska A, Niklinska W, Kozlowski M, Minarowski L, Naumnik W, Niklinski J, Dabrowska K, Chyczewski. Detection of DNA methylation in eucaryotic cells. L Folia Histochem Cytobiol. 2007; 45(4): 315-324.

16. Tunc O, Tremellen K. Oxidative DNA damage impairs global sperm DNA methylation in infertile men. J Assist Reprod Genet. 2009; 26(910): 537-544.

17. Percipalle P. New insights into co-transcriptional sorting of mRNA for cytoplasmic transport during development. Semin Cell Dev Biol. 2014; 32: 55-62.

18. Paradowska-Dogan A, Fernandez A, Bergmann M, Kretzer K, Mallidis C, Vieweg M, Waliszewski P, Zitzmann M, Weidner W, Steger K, Kliesch $\mathrm{S}$. Protamine $\mathrm{mRNA}$ ratio in stallion spermatozoa correlates with mare fecundity. Andrology 2014; 2(4): 521-530.
19. Fritz-Niggli H, Schaeppi-Buechi C.Adaptive response to dominant lethality of mature (class A) and immature (class B) oocytes of D. melanogaster to low doses of ionizing radiation: effects in repairproficient (yw) and repair-deficient strains (mei 41D5 and mus 302D1). Int J Radiat Biol. 1991; 59(1): 175-184.

20. Ashwood-Smith MJ, Edwards RG, DNA repair by oocytes. Mol Hum Reprod. 1996; 2(1): 46-51.

21. Barber RC, Hickenbotham P, Hatch T, Kelly D, Topchiy N, et al. Radiation-induced transgenerational alterations in genome stability and DNA damage. Oncogene 2006; 25(56): 7336-7342.

22. Anway MD, Cupp AS, Uzumcu M, Skinner MK. Epigenetic transgenerational actions of endocrine disruptors and male fertility. Science 2005; 308(5727): 1466-1469.

23. Bufton L, Bruns GA, Magenis RE, Tomar D, Shaw D, Brook D, Litt M. Four restriction fragment length polymorphisms revealed by probes from a single cosmid map to chromosome 19. Am J Hum Genet. 1986; 38(4): 447-460.

24. Fernández-González R, Moreira PN, Pérez-Crespo M, Sánchez-Martín M, Ramirez MA, et al. Long-term effects of mouse intracytoplasmic sperm injection with DNA-fragmented sperm on health and behavior of adult offspring. Biol Reprod. 2008; 78: 761-772.

25. Manicardi GC, Bianchi PG, Pantano S, Azzoni P, Bizzaro D, Bianchi U, Sakkas D.Presence of endogenous nicks in DNA of ejaculated human spermatozoa and its relationship to chromomycin A3 accessibility.Biol Reprod. 1995; 52(4): 864-867.

26. Ji BT, Shu XO, Linet MS, Zheng W, Wacholder S, Gao YT, Ying DM, Jin F. Paternal cigarette smoking and the risk of childhood cancer among offspring of nonsmoking mothers. J Natl Cancer Inst. 1997; 89(3): 238-244.

27. Sorahan T, Lancashire RJ, Hultén MA, Peck I, Stewart AM. Childhood cancer and parental use of tobacco: deaths from 1953 to 1955 . Br J Cancer. 1997; 75(1): 134-138.

28. Wdowiak A, LewickaM, Plewka K, Bakalczuk G. Nicotinism and quality of embryos obtained in in-vitro fertilization programmes. Ann Agric Environ Med. 2013; 20(1): 82-85.

29. Dubrova YE. Radiation-induced transgenerational instability. Oncogene 2003; 22(45): 7087-7093.

30. Mohr U, Dasenbrock C, Tillmann T, Kohler M, Kamino K, Hagemann G, Morawietz G, Campo E, Cazorla M, Fernandez P, Hernandez L, Cardesa A, Tomatis L. Possible carcinogenic effects of X-rays in a transgenerational study with CBA mice. Carcinogenesis 1999; 20(2): 325-332.

31. Chen SJ, Allam JP, Duan YG, Haidl G. Influence of reactive oxygen species on human sperm functions and fertilizing capacity including therapeutical approaches. Arch Gynecol Obstet. 2013; 288(1): 191-199.

32. Mason JB, Dickstein A, Jacques PF, Haggarty P, Selhub J, Dallal G, Rosenberg IH.A temporal association between folic acid fortification and an increase in colorectal cancer rates may be illuminating important biological principles: a hypothesis. Cancer Epidemiol Biomarkers Prev. 2007; 16(7): 1325-1329.

33. Alvarez Sedó C, Barros MA. Uriondo Boudri H. Rougier N, Papier S, Nodar F. Changes in DNA fragmentation during sperm preparation for ICSI over time 109. IBRA Assist Reprod. 2013; 17: 109-114. 